PROCEEDINGS OF THE

AMERICAN MATHEMATICAL SOCIETY

Volume 135, Number 10, October 2007, Pages 3029-3037

S 0002-9939(07)08994-0

Article electronically published on June 19, 2007

\title{
ON THE SUM FORMULA FOR THE $q$-ANALOGUE OF NON-STRICT MULTIPLE ZETA VALUES
}

\author{
YASUO OHNO AND JUN-ICHI OKUDA
}

(Communicated by Jonathan M. Borwein)

\begin{abstract}
In this article, the $q$-analogues of the linear relations of non-strict multiple zeta values called "the sum formula" and "the cyclic sum formula" are established.
\end{abstract}

\section{INTRODUCTION}

For any multi-index $\mathbf{k}=\left(k_{1}, k_{2}, \ldots, k_{r}\right)\left(k_{i} \in \mathbb{Z}, k_{i}>0\right)$, the weight $\operatorname{wt}(\mathbf{k})$ and depth $\operatorname{dep}(\mathbf{k})$ of $\mathbf{k}$ are by definition the integers $k=k_{1}+k_{2}+\cdots+k_{r}$ and $r$ respectively. We denote by $I(k, r)$ the set of multi-indices $\mathbf{k}$ of weight $k$, and depth $r$, and by $I_{0}(k, r)$ the subset of admissible indices, i.e., indices with the extra requirement that $k_{1} \geq 2$.

For an admissible index $\left(k_{1}, \ldots, k_{r}\right)$, the multiple zeta value [23, 11] (MZV, for short) and the non-strict multiple zeta value are defined as follows:

$$
\zeta\left(k_{1}, \ldots, k_{r}\right):=\sum_{n_{1}>\cdots>n_{r}>0} \frac{1}{n_{1}^{k_{1}} \cdots n_{r}^{k_{r}}}, \quad \zeta^{\star}\left(k_{1}, \ldots, k_{r}\right):=\sum_{n_{1} \geq \cdots \geq n_{r} \geq 1} \frac{1}{n_{1}^{k_{1}} \cdots n_{r}^{k_{r}}} .
$$

The latter is also called multiple zeta-star value (MZSV, for short) in 1, 19. Both values can be written as a $\mathbb{Z}$-linear combination of each other.

These values are known to be related to many objects of mathematics and quantum physics, for example, connection formulae for hypergeometric functions [20, knot invariants [16], Feynman diagrams [15] and so on. They also appear in the coefficients of the Drinfel'd's KZ-associator [7]. The properties of the KZ-associator are related to the representations of the fundamental group of a configuration space.

Study of MZSVs has been initiated by Leonhard Euler [ [8], and he got many results including the well-known formula:

$$
\zeta^{\star}(k-1,1)=\frac{k+1}{2} \zeta(k)-\frac{1}{2} \sum_{r=2}^{k-2} \zeta(r) \zeta(k-r) .
$$

Received by the editors March 1, 2006

2000 Mathematics Subject Classification. Primary 11M41, 33D15, 11B65, 05A30, 11M06.

Key words and phrases. Multiple zeta values, non-strict multiple zeta values, multiple zeta star values, sum formula, $q$-analogue, $q$-series, basic hypergeometric function.

The first author was partly supported by Grant-in-Aid for Young Scientists (B) No. 18740020 and the second author was partly supported by Grant-in-Aid for Young Scientists (B) No. 17740026 from the Ministry of Education, Culture, Sports, Science and Technology, Japan.

(C)2007 American Mathematical Society Reverts to public domain 28 years from publication 
It seems that Euler wanted to give an answer to the question, "When are MZSVs in the algebra generated by Riemann zeta values $\zeta(k)$ ?" It is a basic and an important question even now. The next two equivalent formulae were conjectured in [11], and proved by Andrew Granville [10] and Don Zagier independently.

Sum Formula. For positive integers $0<r<k$, there holds

$$
\sum_{\mathbf{k} \in I_{0}(k, r)} \zeta(\mathbf{k})=\zeta(k), \quad \sum_{\mathbf{k} \in I_{0}(k, r)} \zeta^{\star}(\mathbf{k})=\left(\begin{array}{c}
k-1 \\
r-1
\end{array}\right) \zeta(k) .
$$

These formulae are so fundamental that they are re-proved again and again 2, 12, 13, 17, 18, 20, 22, For the MZSV case, there are two more proofs. One is the conclusion of the cyclic sum formula for MZSVs [19]. The other is using the special value of the generating function of multiple polylogarithms [14].

Cyclic Sum Formula. For $\left(k_{1}, \ldots, k_{r}\right) \in I_{0}(k, r)$,

$$
\sum_{i=1}^{r} \sum_{j=0}^{k_{i}-2} \zeta^{\star}\left(k_{i}-j, k_{i+1}, \ldots, k_{r}, k_{1}, \ldots, k_{i-1}, j+1\right)=k \zeta(k+1),
$$

where the empty sum means zero.

Generating Function. For the multiple polylogarithms with equality defined by

$$
\mathrm{Li}_{k_{1}, \ldots, k_{r}}^{\star}(t):=\sum_{n_{1} \geq \cdots \geq n_{r} \geq 1} \frac{t^{n_{1}}}{n_{1}^{k_{1}} \cdots n_{r}^{k_{r}}},
$$

the generating function and its special value at $t=1$ are expressed as follows:

$$
\begin{aligned}
& \sum_{k>r>0}\left\{\sum_{\mathbf{k} \in I_{0}(k, r)} \operatorname{Li}_{\mathbf{k}}^{\star}(t)\right\} x^{k-r-1} y^{r-1} \\
& \quad=\frac{1}{1-x-y} \int_{0}^{t}(1-s)^{-y}{ }_{2} F_{1}(1-x-y, 1-y, 2-x-y ; s) d s, \\
& \sum_{k>r>0}\left\{\sum_{\mathbf{k} \in I_{0}(k, r)} \zeta^{\star}(\mathbf{k})\right\} x^{k-r-1} y^{r-1}=\sum_{n=1}^{\infty} \frac{1}{n-x-y} \frac{1}{n-y},
\end{aligned}
$$

where ${ }_{2} F_{1}$ is Gauß's hypergeometric series.

In this article, we define the $q$-analogues of MZSVs and construct the $q$-analogues of the above formulae.

For $0<q<1$ and $\alpha \in \mathbb{C},[\alpha]$ is defined by $[\alpha]:=\left(1-q^{\alpha}\right) /(1-q) \stackrel{q \rightarrow 1}{\longrightarrow} \alpha$. The $q$-Pochhammer symbol is defined by $(\alpha ; q)_{\infty}:=\prod_{j=0}^{\infty}\left(1-\alpha q^{j}\right)$ and $(\alpha ; q)_{n}:=$ $(\alpha ; q)_{\infty} /\left(\alpha q^{n} ; q\right)_{\infty}$ for any integer $n$. Then the $q$-analogues of MZV are defined by

$$
\zeta_{q}\left[k_{1}, \ldots, k_{r}\right]:=\sum_{n_{1}>\cdots>n_{r}>0} \frac{q^{n_{1}\left(k_{1}-1\right)+\cdots+n_{r}\left(k_{r}-1\right)}}{\left[n_{1}\right]^{k_{1}} \cdots\left[n_{r}\right]^{k_{r}}} .
$$

A $q$-analogue of the relations for MZVs are considered in [3, 5, 6, 21]. A finite $q$-analogue of $\zeta^{\star}(\mathbf{k})$ is studied in [4]. 
Definition 1. For any admissible index $\left(k_{1}, \ldots, k_{r}\right)$, the $q$-analogue of MZSV and the multiple polylogarithm with equality are as follows:

$$
\begin{aligned}
\zeta_{q}^{\star}\left[k_{1}, \ldots, k_{r}\right] & :=\sum_{n_{1} \geq \cdots \geq n_{r} \geq 1} \frac{q^{n_{1}\left(k_{1}-1\right)+\cdots+n_{r}\left(k_{r}-1\right)}}{\left[n_{1}\right]^{k_{1}} \cdots\left[n_{r}\right]^{k_{r}}}, \\
\operatorname{Li}_{k_{1}, \ldots, k_{r}}^{\star}[t]: & =\sum_{n_{1} \geq \cdots \geq n_{r} \geq 1} \frac{t^{n_{1}}}{\left[n_{1}\right]^{k_{1}} \cdots\left[n_{r}\right]^{k_{r}}} .
\end{aligned}
$$

As the $q$-analogue of (2), we have the next formula:

Theorem 1 (Cyclic Sum Formula). For $\left(k_{1}, \ldots, k_{r}\right) \in I_{0}(k, r)$,

$$
\begin{aligned}
\sum_{i=1}^{r} \sum_{j=0}^{k_{i}-2} \zeta_{q}^{\star}\left[k_{i}-j, k_{i+1}, \ldots, k_{r}, k_{1}, \ldots,\right. & \left.k_{i-1}, j+1\right] \\
& =\sum_{l=0}^{r}(k-l)\left(\begin{array}{l}
r \\
l
\end{array}\right)(1-q)^{l} \zeta_{q}[k-l+1],
\end{aligned}
$$

where the empty sum means zero.

Moreover, there also holds the $q$-analogue of (3):

Theorem 2 (Generating Function of Multiple Polylogarithms).

$$
\sum_{k>r>0}\left\{\sum_{\mathbf{k} \in I_{0}(k, r)} \operatorname{Li}_{\mathbf{k}}^{\star}[t]\right\} u^{k-r-1} v^{r-1}=\frac{1}{1-u-v} \int_{0}^{t} \frac{(s ; q)_{\infty}}{(b s ; q)_{\infty}}{ }_{2} \phi_{1}(a, b, a q ; s, q) d_{q} s,
$$

where ${ }_{2} \phi_{1}$ is Heine's q-hypergeometric series $[9], q^{-a-1}=\frac{1}{1-(1-q)(u+v)}$ and $b=$ $\frac{1-(1-q) u}{1-(1-q)(u+v)}$, and the integral is the Jackson q-integral [9]

$$
\int_{0}^{t} f(s) d_{q} s:=(1-q) t \sum_{n=0}^{\infty} f\left(q^{n} t\right) q^{n} .
$$

As the corollary of these theorems, we obtain the $q$-analogue of (1):

Corollary 3 (Sum Formula). For integers $0<r<k$,

$$
\sum_{\mathbf{k} \in I_{0}(k, r)} \zeta_{q}^{\star}[\mathbf{k}]=\frac{1}{k-1}\left(\begin{array}{c}
k-1 \\
r-1
\end{array}\right) \sum_{l=0}^{r-1}\left(\begin{array}{c}
r-1 \\
l
\end{array}\right)(k-1-l)(1-q)^{l} \zeta_{q}[k-l] .
$$

\section{Proof of Theorem 1}

For index $\left(k_{1}, \ldots, k_{r}\right) \in I(k, r)$ with $k_{i} \geq 2$ for some $i$, we set the convergent series

$$
T\left(k_{1}, \ldots, k_{r}\right):=\sum_{\substack{n_{1} \geq \cdots \geq n_{r+1} \geq 1 \\ n_{1} \neq n_{r+1}}} \frac{q^{n_{1}\left(k_{1}-1\right)+\cdots+n_{r}\left(k_{r}-1\right)}}{\left[n_{1}\right]^{k_{1}} \cdots\left[n_{r}\right]^{k_{r}}} \frac{q^{n_{1}-n_{r+1}}}{\left[n_{1}-n_{r+1}\right]} .
$$

This series satisfies the equation

$$
\begin{aligned}
& T\left(k_{1}, k_{2}, \ldots, k_{r}\right)-T\left(k_{2}, \ldots, k_{r}, k_{1}\right) \\
& =\sum_{l=0}^{r}\left(\begin{array}{l}
r \\
l
\end{array}\right)\left(k_{1}-\frac{l}{r}\right)(1-q)^{l} \zeta_{q}[k-l+1]-\sum_{j=0}^{k_{1}-2} \zeta_{q}^{\star}\left[k_{1}-j, k_{2}, \ldots, k_{r}, j+1\right] .
\end{aligned}
$$


Summing up these equations by rotating the indices, and we have the theorem.

To prove (6), by using the equation

$$
\frac{1}{\left[n_{1}\right]} \frac{q^{n_{1}-n_{r+1}}}{\left[n_{1}-n_{r+1}\right]}=\left(\frac{1}{\left[n_{1}-n_{r+1}\right]}-\frac{1}{\left[n_{1}\right]}\right) \frac{1}{\left[n_{r+1}\right]},
$$

we have

$$
\begin{aligned}
& T\left(k_{1}, k_{2}, \ldots, k_{r}\right) \\
& =\sum_{\substack{n_{1} \geq \cdots \geq n_{r+1} \geq 1 \\
n_{1} \neq n_{r+1}}} \frac{q^{n_{1}\left(k_{1}-1\right)+n_{2}\left(k_{2}-1\right)+\cdots+n_{r}\left(k_{r}-1\right)}}{\left[n_{1}\right]^{k_{1}-1}\left[n_{2}\right]^{k_{2}} \cdots\left[n_{r}\right]^{k_{r}}}\left(\frac{1}{\left[n_{1}-n_{r+1}\right]}-\frac{1}{\left[n_{1}\right]}\right) \frac{1}{\left[n_{r+1}\right]} \\
& =\sum_{\substack{n_{1} \geq \cdots \geq n_{r+1} \geq 1 \\
n_{1} \neq n_{r+1}}} \frac{q^{n_{1}\left(k_{1}-2\right)+n_{2}\left(k_{2}-1\right)+\cdots+n_{r}\left(k_{r}-1\right)}}{\left[n_{1}\right]^{k_{1}-1}\left[n_{2}\right]^{k_{2}} \cdots\left[n_{r}\right]^{k_{r}}\left[n_{r+1}\right]^{1}} \frac{q^{n_{1}}}{\left[n_{1}-n_{r+1}\right]} \\
& -\zeta_{q}^{\star}\left[k_{1}, k_{2}, \ldots, k_{r}, 1\right]+\sum_{n=1}^{\infty} \frac{q^{n(k-r)}}{[n]^{k+1}} \\
& =\sum_{\substack{n_{1} \geq \cdots \geq n_{r+1} \geq 1 \\
n_{1} \neq n_{r+1}}} \frac{q^{n_{1}\left(k_{1}-2\right)+n_{2}\left(k_{2}-1\right)+\cdots+n_{r}\left(k_{r}-1\right)}}{\left[n_{1}\right]^{k_{1}-2}\left[n_{2}\right]^{k_{2}} \cdots\left[n_{r}\right]^{k_{r}}\left[n_{r+1}\right]^{1}}\left(\frac{1}{\left[n_{1}-n_{r+1}\right]}-\frac{1}{\left[n_{1}\right]}\right) \frac{q^{n_{r+1}}}{\left[n_{r+1}\right]} \\
& -\zeta_{q}^{\star}\left[k_{1}, k_{2}, \ldots, k_{r}, 1\right]+\sum_{n=1}^{\infty} \frac{q^{n(k-r)}}{[n]^{k+1}} \\
& =\sum_{\substack{n_{1} \geq \cdots \geq n_{r+1} \geq 1 \\
n_{1} \neq n_{r+1}}} \frac{q^{n_{1}\left(k_{1}-3\right)+n_{2}\left(k_{2}-1\right)+\cdots+n_{r}\left(k_{r}-1\right)+n_{r+1}(2-1)}}{\left[n_{1}\right]^{k_{1}-2}\left[n_{2}\right]^{k_{2}} \cdots\left[n_{r}\right]^{k_{r}}\left[n_{r+1}\right]^{2}} \frac{q^{n_{1}}}{\left[n_{1}-n_{r+1}\right]} \\
& -\zeta_{q}^{\star}\left[k_{1}, k_{2}, \ldots, k_{r}, 1\right]-\zeta_{q}^{\star}\left[k_{1}-1, k_{2}, \ldots, k_{r}, 2\right]+2 \sum_{n=1}^{\infty} \frac{q^{n(k-r)}}{[n]^{k+1}} \\
& =\cdots \cdots+\cdots \cdots \\
& =\sum_{\substack{n_{1} \geq \cdots \geq n_{r+1} \geq 1 \\
n_{1} \neq n_{r+1}}} \frac{q^{n_{2}\left(k_{2}-1\right)+\cdots+n_{r}\left(k_{r}-1\right)+n_{r+1}\left(k_{1}-2\right)}}{\left[n_{1}\right]^{1}\left[n_{2}\right]^{k_{2}} \cdots\left[n_{r}\right]^{k_{r}}\left[n_{r+1}\right]^{k_{1}-1}} \frac{q^{n_{1}}}{\left[n_{1}-n_{r+1}\right]} \\
& -\sum_{j=0}^{k_{1}-2} \zeta_{q}^{\star}\left[k_{1}-j, k_{2}, \ldots, k_{r}, j+1\right]+\left(k_{1}-1\right) \sum_{n=1}^{\infty} \frac{q^{n(k-r)}}{[n]^{k+1}} .
\end{aligned}
$$

By using the equation

$$
\frac{1}{\left[n_{1}\right]} \frac{q^{n_{1}}}{\left[n_{1}-n_{r+1}\right]}=\left(\frac{q^{n_{1}-n_{r+1}}}{\left[n_{1}-n_{r+1}\right]}-\frac{q^{n_{1}}}{\left[n_{1}\right]}\right) \frac{q^{n_{r+1}}}{\left[n_{r+1}\right]},
$$


the first term is

$$
\begin{aligned}
& \sum_{\substack{n_{1} \geq \cdots \geq n_{r+1} \geq 1 \\
n_{1} \neq n_{r+1}}} \frac{q^{n_{2}\left(k_{2}-1\right)+\cdots+n_{r}\left(k_{r}-1\right)+n_{r+1}\left(k_{1}-2\right)}}{\left[n_{1}\right]^{1}\left[n_{2}\right]^{k_{2}} \cdots\left[n_{r}\right]^{k_{r}\left[n_{r+1}\right]^{k_{1}-1}}} \frac{q^{n_{1}}}{\left[n_{1}-n_{r+1}\right]} \\
& =\sum_{\substack{n_{2} \geq \cdots \geq n_{r+1} \geq 1 \\
n_{2} \neq n_{r+1}}} \frac{q^{n_{2}\left(k_{2}-1\right)+\cdots+n_{r}\left(k_{r}-1\right)+n_{r+1}\left(k_{1}-2\right)}}{\left[n_{2}\right]^{k_{2} \cdots\left[n_{r}\right]^{k_{r}}\left[n_{r+1}\right]^{k_{1}-1}}} \\
& \times\left(\sum_{n_{1}=n_{2}}^{\infty} \frac{q^{n_{1}-n_{r+1}}}{\left[n_{1}-n_{r+1}\right]}-\frac{q^{n_{1}}}{\left[n_{1}\right]}\right) \frac{q^{n_{r+1}}}{\left[n_{r+1}\right]} \\
& +\sum_{n_{2}=1}^{\infty} \frac{q^{n_{2}\left(k_{2}+\cdots+k_{r}+k_{1}-r\right)}}{\left[n_{2}\right]^{k_{2}+\cdots+k_{r}+k_{1}}}\left(\sum_{n_{1}=n_{2}+1}^{\infty} \frac{q^{n_{1}-n_{2}}}{\left[n_{1}-n_{2}\right]}-\frac{q^{n_{1}}}{\left[n_{1}\right]}\right) \\
& =\sum_{\substack{n_{2} \geq \cdots \geq n_{r+2} \geq 1 \\
n_{2} \neq n_{r+2}}} \frac{q^{n_{2}\left(k_{2}-1\right)+\cdots+n_{r}\left(k_{r}-1\right)+n_{r+1}\left(k_{1}-1\right)}}{\left[n_{2}\right]^{k_{2}} \cdots\left[n_{r}\right]^{k_{r}}\left[n_{r+1}\right]^{k_{1}}} \frac{q^{n_{2}-n_{r+2}}}{\left[n_{2}-n_{r+2}\right]}+\sum_{n=1}^{\infty} \frac{q^{n(k-r+1)}}{[n]^{k+1}} .
\end{aligned}
$$

Moreover, by substituting the equation

$$
\sum_{n=1}^{\infty} \frac{q^{n(k-m)}}{[n]^{k+1}}=\sum_{l=0}^{m}\left(\begin{array}{c}
m \\
l
\end{array}\right)(1-q)^{l} \zeta_{q}[k-l+1]
$$

we obtain (6).

Furthermore, for $\mathbf{k}=\left(k_{1}, \ldots, k_{r}\right) \in I_{0}(k, r)$, we set

$$
J_{0}(\mathbf{k}):=\bigcup_{i=1}^{r} \bigcup_{j=0}^{k_{i}-2}\left\{\left(k_{i}-j, k_{i+1}, \ldots, k_{r}, k_{1}, \ldots, k_{i-1}, j+1\right)\right\} \subset I_{0}(k+1, r+1) .
$$

Then

$$
I_{0}(k+1, r+1)=\bigcup_{\mathbf{k} \in I_{0}(k, r)} J_{0}(\mathbf{k}), \quad \text { and } \quad J_{0}(\mathbf{k}) \cap J_{0}\left(\mathbf{k}^{\prime}\right)=\emptyset \quad \text { if } \mathbf{k} \neq \mathbf{k}^{\prime} .
$$

From (4) we have

$$
\sum_{\mathbf{k}^{\prime} \in J_{0}(\mathbf{k})} \zeta_{q}^{\star}\left[\mathbf{k}^{\prime}\right]=\frac{\# J_{0}(\mathbf{k})}{k-r} \sum_{l=0}^{r}(k-l)\left(\begin{array}{l}
r \\
l
\end{array}\right)(1-q)^{l} \zeta_{q}[k-l+1],
$$

and summing up about $\mathbf{k}$ we obtain

$$
\begin{aligned}
\sum_{\mathbf{k}^{\prime \prime} \in I_{0}(k+1, r+1)} \zeta_{q}^{\star}\left[\mathbf{k}^{\prime \prime}\right] & =\sum_{\mathbf{k} \in I_{0}(k, r)} \sum_{\mathbf{k}^{\prime} \in J_{0}(\mathbf{k})} \zeta_{q}^{\star}\left[\mathbf{k}^{\prime}\right] \\
& =\frac{\# I_{0}(k+1, r+1)}{k-r} \sum_{l=0}^{r}(k-l)\left(\begin{array}{c}
r \\
l
\end{array}\right)(1-q)^{l} \zeta_{q}[k-l+1] \\
& =\frac{1}{k-r}\left(\begin{array}{c}
k-1 \\
r
\end{array}\right) \sum_{l=0}^{r}(k-l)\left(\begin{array}{c}
r \\
l
\end{array}\right)(1-q)^{l} \zeta_{q}[k-l+1] .
\end{aligned}
$$

Thus we have Corollary 3 , 


\section{Proof of Theorem 2}

We denote the generating functions of $\mathrm{Li}_{\mathbf{k}}^{\star}$ as follows:

$$
\left\{\begin{array}{l}
\Psi^{\star}(u, v ; t, q):=\sum_{k \geq r>0}\left(\sum_{\mathbf{k} \in I(k, r)} \mathrm{Li}_{\mathbf{k}}^{\star}[t]\right) u^{k-r} v^{r-1}, \\
\Psi_{0}^{\star}(u, v ; t, q):=\sum_{k>r>0}\left(\sum_{\mathbf{k} \in I_{0}(k, r)} \operatorname{Li}_{\mathbf{k}}^{\star}[t]\right) u^{k-r-1} v^{r-1} .
\end{array}\right.
$$

To investigate the above generating functions we use the $q$-differential equation, where the $q$-differential operator $D_{q}$ is defined by

$$
\left(D_{q} f\right)(t):=\frac{f(t)-f(q t)}{t-q t} .
$$

From the $q$-differential equation for $\mathrm{Li}_{\mathbf{k}}^{\star}$

$$
D_{q} \mathrm{Li}_{k_{1}, k_{2}, \ldots, k_{r}}^{\star}[t]= \begin{cases}\frac{1}{t} \operatorname{Li}_{k_{1}-1, k_{2}, \ldots, k_{r}}^{\star}[t] & \left(k_{1} \geq 2\right), \\ \frac{1}{t} \frac{1}{1-t} \mathrm{Li}_{k_{2}, \ldots, k_{r}}^{\star}[t] & \left(k_{1}=1 \text { and } r \geq 2\right), \\ \frac{1}{1-t} & \left(k_{1}=1 \text { and } r=1\right),\end{cases}
$$

$\Psi^{\star}$ and $\Psi_{0}^{\star}$ satisfy the following $q$-differential equations:

$$
\left\{\begin{aligned}
D_{q} \Psi_{0}^{\star}(u, v ; t, q) & =\frac{1}{t} \Psi^{\star}(u, v ; t, q), \\
D_{q}\left(\Psi^{\star}-u \Psi_{0}^{\star}\right)(u, v ; t, q) & =\frac{1}{1-t}+\frac{1}{t} \frac{1}{1-t} v \Psi^{\star}(u, v ; t, q) .
\end{aligned}\right.
$$

By eliminating $\Psi^{\star}$ from the above equations, we have that $\Psi_{0}^{\star}$ satisfies the inhomogeneous linear $q$-differential equation of second order:

$$
q t(1-t) D_{q}^{2} f+\{(1-t)(1-u)-v\} D_{q} f=1
$$

$\Psi_{0}^{\star}$ is characterized as the regular solution of (7) around the origin and the value at the origin is 0 .

We must find such a solution of (7) in another way. At first we put $g:=D_{q} f$ and solve the equation

$$
q t(1-t) D_{q} g+\{(1-t)(1-u)-v\} g=1,
$$

by variation of parameter. We choose $C_{0} t^{a}(t ; q)_{\infty} /(b t ; q)_{\infty}$ for the solution of the homogeneous equation

$$
q t(1-t) D_{q} h+\{(1-t)(1-u)-v\} h=0,
$$

where $q^{-a-1}=\frac{1}{1-(1-q)(u+v)}, b=\frac{1-(1-q) u}{1-(1-q)(u+v)}$ and $C_{0} \in \mathbb{C}$. We assume that

$$
g(t)=C(t) t^{a} \frac{(t ; q)_{\infty}}{(b t ; q)_{\infty}},
$$

and substitute this into (8); then we have

$$
C^{\prime}(t)=q^{-a-1} t^{-a-1} \frac{(b q t ; q)_{\infty}}{(t ; q)_{\infty}}
$$


The Jackson integral of $C^{\prime}(t)$ is as follows:

$$
\begin{aligned}
\int_{0}^{t} q^{-a-1} s^{-a-1} \frac{(b q s ; q)_{\infty}}{(s ; q)_{\infty}} d_{q} s & =q^{-a-1} \int_{0}^{t} s^{-a-1} \sum_{n=0}^{\infty} \frac{(b q ; q)_{n}}{(q ; q)_{n}} s^{n} d_{q} s \\
& =q^{-a-1} \sum_{n=0}^{\infty} \frac{(b q ; q)_{n}}{(q ; q)_{n}} \frac{t^{n-a}}{[n-a]} \\
& =\frac{t^{-a}}{1-u-v^{2}} \phi_{1}\left(q^{-a}, b q, q^{-a+1} ; t, q\right)
\end{aligned}
$$

where the first equality is by virtue of the $q$-binomial theorem $[9$. So we obtain the solution of (8) which is regular at the origin:

$$
g(t)=\frac{1}{1-u-v} \frac{(t ; q)_{\infty}}{(b t ; q)_{\infty}}{ }_{2} \phi_{1}\left(q^{-a}, b q, q^{-a+1} ; t, q\right)
$$

We consider the Jackson integral again and get the solution of (7):

$$
f(t)=\frac{1}{1-u-v} \int_{0}^{t} \frac{(s ; q)_{\infty}}{(b s ; q)_{\infty}}{ }_{2} \phi_{1}\left(q^{-a}, b q, q^{-a+1} ; s, q\right) d_{q} s
$$

By executing the Jackson integral, we have

$$
\begin{aligned}
f(t) & =\frac{1}{1-u-v} \sum_{n=0}^{\infty} \frac{\left(1-q^{-a}\right)(b q ; q)_{n}}{\left(1-q^{n-a}\right)(q ; q)_{n}} \int_{0}^{t} s^{n} \frac{(s ; q)_{\infty}}{(b s ; q)_{\infty}} d_{q} s \\
& =\frac{1}{1-u-v} \sum_{n=0}^{\infty} \frac{\left(1-q^{-a}\right)(b q ; q)_{n}}{\left(1-q^{n-a}\right)(q ; q)_{n}}(1-q) t^{n+1} \frac{(t)_{\infty}}{(b t)_{\infty}} \sum_{j=0}^{\infty} q^{j(n+1)} \frac{(b t)_{j}}{(t)_{j}}
\end{aligned}
$$

which is zero at $t=0$. Thus we obtain the theorem.

In the same way as [21], the special value of $\mathrm{Li}^{\star}$ and the generating function are expressed by the combination of the $q$-analogue of MZSVs : Substitute $t=q$ and the value is

$$
\begin{aligned}
\mathrm{Li}_{k_{1}, k_{2}, \ldots, k_{r}}^{\star}[q]=\sum_{a_{1}=0}^{k_{1}-2} \sum_{a_{2}=0}^{k_{2}-1} \cdots \sum_{a_{r}=0}^{k_{r}-1}\left(\begin{array}{c}
k_{1}-2 \\
a_{1}
\end{array}\right)\left(\begin{array}{c}
k_{2}-1 \\
a_{2}
\end{array}\right) \cdots\left(\begin{array}{c}
k_{r}-1 \\
a_{r}
\end{array}\right) \\
\times(1-q)^{k_{1}+\cdots+k_{r}-a_{1}-\ldots-a_{r}} \zeta_{q}^{\star}\left[a_{1}, a_{2}, \ldots, a_{r}\right]
\end{aligned}
$$

and the generating function is

$$
\Psi^{\star}(u, v ; q, q)=\frac{1}{1+(1-q) x} \sum_{k>r>0}\left\{\sum_{\mathbf{k} \in I_{0}(k, r)} \zeta_{q}^{\star}[\mathbf{k}]\right\} x^{k-r-1} y^{r-1},
$$

where $x=\frac{u}{1-(1-q) u}$ and $y=\frac{v}{1-(1-q) u}$. 
On the other hand, by substituting $t=q$ to (9),

$$
\begin{aligned}
\Psi^{\star}(u, v ; q, q)= & \frac{1}{1-u-v} \sum_{n=0}^{\infty} \frac{\left(1-q^{-a}\right)(b q ; q)_{n}}{\left(1-q^{n-a}\right)(q ; q)_{n}}(1-q) q^{n+1} \frac{(q ; q)_{\infty}}{(b q ; q)_{\infty}} \\
& \times \sum_{j=0}^{\infty} q^{j(n+1)} \frac{(b q ; q)_{j}}{(q ; q)_{j}} \\
= & \frac{1-q}{1-u-v} \sum_{n=0}^{\infty} \frac{\left(1-q^{-a}\right)(b q ;)_{n}}{\left(1-q^{n-a}\right)(q ; q)_{n}} q^{n+1} \frac{(q ; q)_{\infty}}{(b q ; q)_{\infty}} \frac{\left(b q^{n+2} ; q\right)}{\left(q^{n+1} ; q\right)} \\
= & \frac{1-q}{1-u-v} \sum_{n=0}^{\infty} \frac{\left(1-q^{-a}\right)}{\left(1-q^{n-a}\right)} \frac{q^{n+1}}{\left(1-b q^{n+1}\right)} \\
= & \sum_{n=1}^{\infty} \frac{q^{n}}{[n]-(u+v)} \frac{1-(1-q)(u+v)}{[n]-\left(1-q^{n}\right) u-v} \\
= & \frac{1}{1+(1-q) x} \sum_{n=1}^{\infty} \frac{q^{n}(1-(1-q) y)}{([n]-y)\left([n]-x q^{n}-y\right)} .
\end{aligned}
$$

Hence we have

$$
\sum_{k>r>0}\left\{\sum_{\mathbf{k} \in I_{0}(k, r)} \zeta_{q}^{\star}[\mathbf{k}]\right\} x^{k-r-1} y^{r-1}=\sum_{n=1}^{\infty} \frac{q^{n}(1-(1-q) y)}{([n]-y)\left([n]-x q^{n}-y\right)},
$$

and expanding the right hand by geometric series, we obtain Corollary 3.

\section{ACKNOWLEDGMENTS}

The authors express their deep gratitude to Professor Yoshihiro Takeyama for his helpful suggestions.

\section{REFERENCES}

1. Takashi Aoki and Yasuo Ohno, Sum relations for multiple zeta values and connection formulas for the Gauss hypergeometric functions, Publ. Res. Inst. Math. Sci. 41 (2005), no. 2, 329-337. MR2138027 (2005m:11165)

2. Jonathan Borwein, David Bailey, and Roland Girgensohn, Experimentation in mathematics, A K Peters Ltd., Natick, MA, 2004, Computational paths to discovery. MR 2051473 (2005h:11002)

3. David M. Bradley, On the sum formula for multiple q-zeta values, Rocky Mountain J. Math. (to appear).

4. _ Duality for finite multiple harmonic q-series, Discrete Math. 300 (2005), no. 1-3, 44-56. MR 2170113 (2006m:05019)

5. _ Multiple q-zeta values, J. Algebra 283 (2005), no. 2, 752-798. MR 2111222 (2006f:11106)

6. , A q-analog of Euler's decomposition formula for the double zeta function, Int. J. Math. Math. Sci. (2005), no. 21, 3453-3458. MR2206867 (2006k:11174)

7. V. G. Drinfel'd, On quasitriangular quasi-Hopf algebras and on a group that is closely connected with $\operatorname{Gal}(\overline{\mathbb{Q}} / \mathbb{Q})$, Algebra i Analiz 2 (1990), no. 4, 149-181. MR1080203 (92f:16047)

8. Leonhard Euler, Meditationes circa singulare serierum genus, Novi Comm. Acad. Sci. Petropol. 20 (1775), 140-186, Reprinted in "Opera Omnia," ser. I, 15, B. G. Teubner, Berlin, 1927, pp. 217-267.

9. George Gasper and Mizan Rahman, Basic hypergeometric series, second ed., Encyclopedia of Mathematics and its Applications, vol. 96, Cambridge University Press, Cambridge, 2004, With a foreword by Richard Askey. MR2128719 (2006d:33028) 
10. Andrew Granville, A decomposition of Riemann's zeta-function, Analytic number theory (Kyoto, 1996), London Math. Soc. Lecture Note Ser., vol. 247, Cambridge Univ. Press, Cambridge, 1997, pp. 95-101. MR1694987 (2000c:11134)

11. Michael E. Hoffman, Multiple harmonic series, Pacific J. Math. 152 (1992), no. 2, 275-290. MR.1141796 (92i:11089)

12. Michael E. Hoffman and Yasuo Ohno, Relations of multiple zeta values and their algebraic expression, J. Algebra 262 (2003), no. 2, 332-347. MR.1971042 (2004c:11163)

13. Kentaro Ihara, Masanobu Kaneko, and Don Zagier, Derivation and double shuffle relations for multiple zeta values, Compos. Math. 142 (2006), no. 2, 307-338. MR2218898

14. Yasuhiro Kombu, Multiple zeta values and hypergeometric differential equations, Master's thesis, Kinki University, 2003.

15. Dirk Kreimer, Knots and Feynman diagrams, Cambridge Lecture Notes in Physics, vol. 13, Cambridge University Press, Cambridge, 2000. MR.1778151 (2002f:81078)

16. Tu Quoc Thang Le and Jun Murakami, Kontsevich's integral for the Homfly polynomial and relations between values of multiple zeta functions, Topology Appl. 62 (1995), no. 2, 193-206. MR.1320252 (96c:57017)

17. Hiroyuki Ochiai, unpublished manuscript, 1997.

18. Yasuo Ohno, A generalization of the duality and sum formulas on the multiple zeta values, J. Number Theory 74 (1999), no. 1, 39-43. MR.1670544 (99k:11138)

19. Yasuo Ohno and Noriko Wakabayashi, Cyclic sum of multiple zeta values, Acta Arith. 123 (2006), no. 3, 289-295. MR2263259

20. Yasuo Ohno and Don Zagier, Multiple zeta values of fixed weight, depth, and height, Indag. Math. (N.S.) 12 (2001), no. 4, 483-487. MR1908876 (2003e:11094)

21. Jun-ichi Okuda and Yoshihiro Takeyama, On relations for the q-multiple zeta values, to appear in Ramanujan Journal, preprint, 2004.

22. Jun-ichi Okuda and Kimio Ueno, Relations for multiple zeta values and Mellin transforms of multiple polylogarithms, Publ. Res. Inst. Math. Sci. 40 (2004), no. 2, 537-564. MR2049646 (2005f:11199)

23. Don Zagier, Values of zeta functions and their applications, First European Congress of Mathematics, Vol. II (Paris, 1992), Progr. Math., vol. 120, Birkhäuser, Basel, 1994, pp. 497512. MR1341859 (96k:11110)

Department of Mathematics, Kinki University, Higashi-Osaka 577-8502, Japan

Current address: Max-Planck-Institute für Mathematik, Vivatsgasse 7, 53111 Bonn, Germany

E-mail address: ohno@math.kindai.ac.jp

Department of Mathematical Sciences, Science and Engineering, Waseda University, TOKYO 169-8555, JAPAN

E-mail address: okuda@gm.math.waseda.ac.jp 